\title{
Obstructive sleep apnea: A wake-up call for better outcomes
}

$F$ or too many of us, a good night's sleep is a rare occurrence. Lack of quality sleep has profound negative effects on our health, safety, and wellbeing. An estimated 50 to 70 million Americans have sleep disturbances, including 10\% to $17 \%$ of men and $3 \%$ to $9 \%$ of women with moderate to severe obstructive sleep apnea (OSA). ${ }^{1}$ Not only is OSA highly prevalent, $82 \%$ to $93 \%$ of individuals with moderate to severe OSA are unaware they have it, and it remains undiagnosed. ${ }^{2}$

OSA is a potentially serious medical disorder affecting the heart, brain, and metabolism. These physiological changes negatively impact public safety, occupational and academic achievement, and even mortality.

This Cleveland Clinic Journal of Medicine supplement presents a state-of-the-art review of OSA, including the health and societal consequences of OSA and current treatment options. The goal of this publication is to inform and educate healthcare providers from all backgrounds and levels of care who are interested in improving patient outcomes through attention to sleep medicine.

Because OSA is prevalent and underdiagnosed, Jessica Vensel Rundo, MD, MS, reviews the symptoms of OSA, clinical presentation, and the readily available, effective screening tools for detecting sleep apnea. Greater awareness and screening for sleep disturbances informs the need for further diagnostic tests such as laboratory polysomnography and home sleep apnea testing.

The link between OSA and the heart is presented by Reena Mehra, MD, MS, with an overview of the physiology of sleep-heart interactions and the association of OSA and cardiovascular health. Dr. Mehra also reviews central sleep apnea and discusses 2 newer therapies for it: adaptive servoventilation and phrenic nerve stimulation.

Dr. Foldvary-Schaefer reported no financial interests or relationships that pose a potential conflict of interest with this article.

doi:10.3949/ccjm.86.s1.01
Beyond heart health, OSA also adversely affects quality of life, safety, and other important health factors. Harneet Walia, MD, discusses consequences of sleep apnea such as daytime sleepiness, fatigue, drowsy driving, depression, metabolic diseases, and cognitive impairment.

Several treatment options exist for patients diagnosed with OSA. Positive airway pressure (PAP) therapy is the gold standard for treatment of OSA. Colleen G. Lance, MD, reviews and presents case scenarios about the efficacy of PAP therapy, features of continuous PAP therapy, and innovative strategies to improve adherence to therapy.

In addition to PAP therapy, there are alternative treatments for OSA that may benefit some patients. Tina Waters, MD, considers alternatives to PAP therapy, such as lifestyle changes, expiratory PAP therapy, oral appliances, upper airway surgery, and hypoglossal nerve stimulation.

I hope you enjoy this supplement and find it useful to improving the health and quality-of-life outcomes of patients in your care.

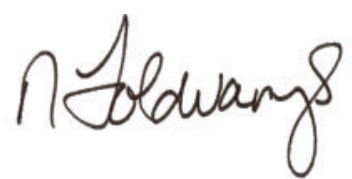

Nancy Foldvary-Schaefer, DO, MS Director, Sleep Medicine Program Neurological Institute Cleveland Clinic

\section{REFERENCES}

1. Peppard PE, Young T, Barnet JH, Palta M, Hagen EW, Hla KM. Increased prevalence of sleep-disordered breathing in adults. Am J Epidemiol 2013; 177(9):1006-1014.

2. Young T, Evans L, Finn L, Palta M. Estimation of the clinically diagnosed proportion of sleep apnea syndrome in middle-aged men and women. Sleep 1997; 20(9):705706. 